\title{
Comparing withdrawal and non-withdrawal of life-sustaining treatment among patients who died from stroke
}

This article was published in the following Dove Press journal:

Vascular Health and Risk Management

3 September 2015

Number of times this article has been viewed

\author{
Eirik Helvig' \\ Lars Thomassen ${ }^{2}$ \\ Ulrike Waje-Andreassen ${ }^{2}$ \\ Halvor Naess ${ }^{3}$ \\ 'Department of Neurology, Haukeland \\ University Hospital, Jonas Liesgt, \\ Bergen, ${ }^{2}$ Institute of Clinical Medicine, \\ University of Bergen, Bergen, ${ }^{3}$ Centre \\ for Age-related Medicine, Stavanger \\ University Hospital, Stavanger, \\ Norway
}

Background: In severe stroke, a decision to withdraw life-sustaining treatment is sometimes made in cooperation with the family. The aim of this study was to study the time from withdrawing life-sustaining treatment to death in patients with severe ischemic or hemorrhagic stroke.

Methods: In total, 2,506 patients with stroke admitted to Haukeland University Hospital between 2006 and 2011 were prospectively registered in the Bergen NORSTROKE database. Risk factors, stroke severity, etiology, and blood analyses were registered. Retrospectively, the patients' records were examined to determine the number of days from withdrawing all life-sustaining treatment to death in patients who died from severe stroke during the hospital stay.

Results: Life-sustaining treatment was withheld in 50 patients with severe stroke. Median time to death after withdrawing life-sustaining treatment was 4 days, and a quarter lived at least 1 week (range $=1-11$ days). Cox regression analyses showed that short time from withdrawing lifesustaining treatment to death was associated with high age (Hazard ratio $[\mathrm{HR}]=1.05, P=0.07$ ), male sex $(\mathrm{HR}=2.9, P=0.01)$, high $\mathrm{C}$-reactive protein on admission $(\mathrm{HR}=1.01, P=0.001)$, and hemorrhagic stroke (versus ischemic stroke, $\mathrm{HR}=1.5, P=0.03$ ).

Conclusion: One week after withdrawing life-sustaining treatment, a quarter of our patients with severe stroke remained alive. Short time to death was associated with high age, male sex, hemorrhagic stroke, and high C-reactive protein on admittance.

Keywords: stroke, withdrawal of life-sustaining treatment, prognosis

\section{Introduction}

In-hospital mortality occurs among one-third of the patients with hemorrhagic stroke $\mathrm{e}^{1,2}$, and in-hospital mortality for ischemic stroke varies from $3 \%$ to $9 \% .^{2-6}$ The percentage of patients with severe stroke has possibly increased in recent years. ${ }^{7}$ Factors associated with early mortality include stroke severity, age, previous stroke, atrial fibrillation, diabetes mellitus, and pneumonia. ${ }^{1,4,6}$

It is the routine in our department to discuss treatment options thoroughly with the patient's family when the stroke is severe. In some cases, when the prognosis is considered to be poor, a joint decision is made to withdraw all life-sustaining treatments including saline and nutrition and only provide alleviating treatment such as morphine and diazepam. These patients have persistent reduction of consciousness and are unable to eat or drink orally. Reliable evidence is needed when making these decisions, and more research on this topic is requested. ${ }^{4}$

The aim of the present retrospective study was to investigate the number of days from withdrawal of life-sustaining treatment to death in patients with severe stroke.
Correspondence: Halvor Naess Department of Neurology, Haukeland University Hospital, Jonas Liesgt, 502I Bergen, Norway Email haln@haukeland.no 


\section{Methods}

All consecutive patients with acute cerebral infarction and cerebral hemorrhage (the index stroke) admitted to the Stroke Unit, Department of Neurology, Haukeland University Hospital between February 2006 and February 2011 were prospectively registered in a database (The Bergen NORSTROKE Registry). Stroke was defined in accordance with the World Health Organization's definition of stroke. ${ }^{8}$ Stroke due to trauma or sinus venous thrombosis were excluded. All had CT and/or MRI. Exact time of onset of stroke, admission, and death were registered. The National Institute of Health Stroke Scale (NIHSS) was used to assess stroke severity on admission.

Traditional risk factors were registered: angina pectoris, myocardial infarction, intermittent claudication, hypertension, diabetes mellitus, and smoking. Current smoking was defined as smoking at least one cigarette per day. Diabetes mellitus was considered present if the patient was on glucose-lowering diet or medication. Hypertension, angina pectoris, myocardial infarction, and peripheral artery disease were considered present if diagnosed by a physician any time before the onset of stroke. Blood analyses including C-reactive protein (CRP) were performed on admission.

For some patients, a decision to withdraw life-sustaining treatment is routinely performed after evaluation of prognosis with the family. These patients suffer from severe deficits including reduced consciousness and inability to communicate. The decision was made on weekdays after summoning the family to a meeting in the hospital. Retrospectively, the patients' records (electronic patient journal) were examined by one of the authors $(\mathrm{EH})$ to determine the number of days from withdrawing all life-sustaining treatment to death because of poor prognosis after a written joint decision with the family. Life-sustaining treatment was defined as the provision of water and nutrition or antibiotics in case of infection. Patients without life-sustaining treatment usually received alleviating treatment, which included morphine $2.5 \mathrm{mg}$ subcutaneously four times daily or more.

Poor prognosis was not defined, but poor prognosis was considered present in patients with stroke with reduced consciousness and high probability of dying irrespective of treatment due to large cerebral infarction or hemorrhage. Patients with poor prognosis were unable to communicate and unable to eat and drink orally.

Additional analyses were done including all patients who died during the hospital stay. Comparisons were performed between patients who died with or without withdrawal of life-sustaining treatment.
The study was approved by the local ethics committee, REK Vest.

\section{Statistics}

Student's $t$-test, pair-wise correlation, chi-square, and Cox regression analyses were used when appropriate. $P$-value $<0.05$ was considered statistically significant. Analyses were carried out using STATA 13.1 (StataCorp LP, College Station, TX, USA).

\section{Results}

\section{Patients who died after withdrawing life-sustaining treatment}

Retrospectively, time for withdrawing life-sustaining treatment was identified in the patient records for 50 patients: 34 patients with ischemic stroke and 16 patients with hemorrhagic stroke. Median NIHSS score on admission was 27 (inter-quartile range $[\mathrm{IQR}]=20-30$ ). In comparison, the median NIHSS score was 3 (IQR $=1-8$ ) among patients who were discharged alive. The location was more frequent in the anterior than the posterior circulation territory for patients with cerebral infarction who died after withdrawing lifesustaining treatment compared with surviving patients ( $94 \%$ vs $80 \%, P=0.04)$. The mean number of days from onset of stroke to death was 9.8 days ( $\mathrm{SD}=6.7$ ), the median 8 days (IQR $=5-14$ ), and the range 2-30 days. The mean number of days from withdrawing all life-sustaining treatments to death was 4.7 days ( $\mathrm{SD}=2.9)$, and the range was $1-11$ days. Table 1 shows percentiles for the number of days from withdrawing life-sustaining treatment to death. The mean number of days from the onset of stroke to withdrawing life-sustaining treatment was 5.0 days $(\mathrm{SD}=5.7)$, the median was 3 days (IQR $=1-8$ ), and the range was 0-26 days.

Figure 1 shows Kaplan-Meier curves for days from withdrawing life-sustaining treatment to death for patients with ischemic stroke and hemorrhagic stroke separately.

Cox regression analyses showed that short time from withdrawing life-sustaining treatment to death was associated

Table I Percentiles and days since withdrawing life-sustaining treatment to death in patients with stroke

\begin{tabular}{ll}
\hline Percentiles & Number of days \\
\hline Smallest & 1 \\
$10 \%$ & 1 \\
$25 \%$ & 2 \\
Median & 4 \\
$75 \%$ & 7 \\
$90 \%$ & 9 \\
Largest & 11 \\
\hline
\end{tabular}




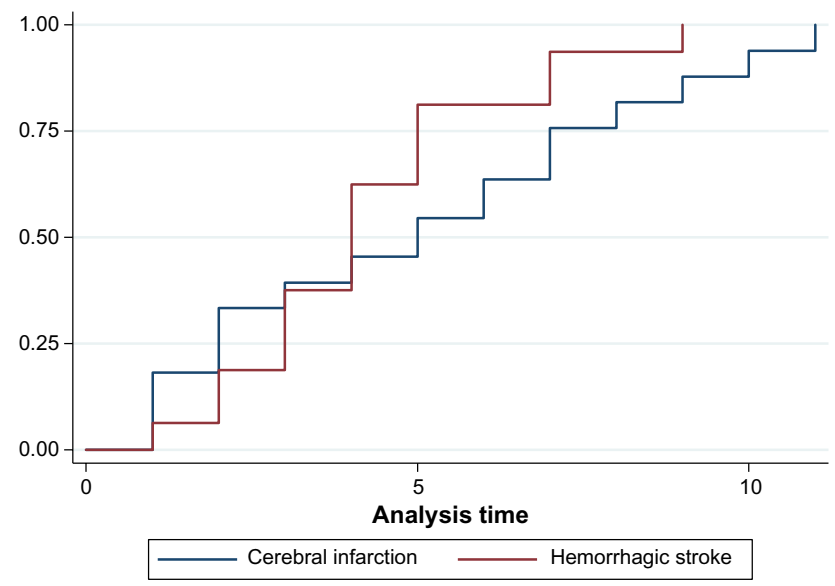

Figure I Kaplan-Meier curves showing days from withdrawing life-sustaining treatment to death in 50 patients with severe cerebral infarction or hemorrhagic stroke $(P=0.20)$.

with high age (Hazard ratio $[\mathrm{HR}]=1.05, P=0.07)$, male sex $(\mathrm{HR}=2.9, P=0.01)$, high $\mathrm{CRP}$ on admission $(\mathrm{HR}=1.01$, $P=0.001$ ), and hemorrhagic stroke (versus ischemic stroke) ( $\mathrm{HR}=1.5, P=0.03$ ). The number of days from withdrawal of life-sustaining treatment to death was not associated with the number of days from the onset of stroke to withdrawal of life-sustaining treatment $(\mathrm{HR}=0.98, P=0.4)$.

In total, 43 (86\%) patients received morphine as alleviating treatment after withdrawing life-sustaining treatment.

\section{Comparing withdrawal and non- withdrawal of life-sustaining treatment among patients who died}

Of 2,506 patients with stroke, 2,217 (88\%) had ischemic stroke and 289 (12\%) had hemorrhagic stroke. Among patients with ischemic stroke, 118 (5.3\%) died, and among patients with hemorrhagic stroke, 66 (23\%) died during the hospital stay. Among patients who died, withdrawal of life-sustaining treatment was associated with higher age and longer time till death (Table 2).

\section{Discussion}

The main finding in our study was that the median time from withdrawing life-sustaining treatment to death in patients with severe stroke was 4 days. Although 4 days is a relatively short period, it is important to recognize that about a quarter of the patients lived a week or longer. All died within 11 days of withdrawing life-sustaining treatment, and the majority died within a few days in accordance with the poor prognosis of these patients. There was very little doubt among the physicians about the poor prognosis among these patients before withdrawing life-sustaining treatment. This knowledge may
Table 2 Characteristics of patients who died in hospital after withdrawal or non-withdrawal of life-sustaining treatment

\begin{tabular}{llll}
\hline & $\begin{array}{l}\text { Withdrawal } \\
\text { N=50 (\%) }\end{array}$ & $\begin{array}{l}\text { No withdrawal } \\
\mathbf{N = 1 3 4}(\%)\end{array}$ & $P$ \\
\hline Male & $18(36)$ & $64(48)$ & 0.15 \\
Female & $32(64)$ & $70(52)$ & - \\
Age (SD) & $83(9)$ & $78(13)$ & 0.03 \\
NIHSS score on & $22(8)$ & $20(8)$ & 0.09 \\
admission (SD) & & & \\
Days to death (SD) & $10(7)$ & $6(8)$ & 0.01 \\
Ischemic stroke & $34(68)$ & $84(63)$ & 0.50 \\
Hemorrhagic stroke & $16(32)$ & $50(37)$ & - \\
Glucose (SD) & $7.3(2.1)$ & $8.5(3.1)$ & 0.01 \\
Diabetes mellitus & $7(14)$ & $25(19)$ & 0.45 \\
C-reactive protein & $24(44)$ & $28(54)$ & 0.68 \\
Hypertension & $22(44)$ & $65(49)$ & 0.59 \\
Atrial fibrillation & $20(40)$ & $57(43)$ & 0.76 \\
\hline
\end{tabular}

Abbreviations: NIHSS, National Institute of Health Stroke Scale; SD, standard deviation.

be helpful for family and caretakers in order to cope with the period after withdrawing life-sustaining treatment. For comparison, only two of 110 patients in an intensive care unit lived more than 7 days after withdrawing life-sustaining treatment. ${ }^{9}$ However, the majority of those patients were weaned off positive-pressure ventilation as part of the procedure to withdraw life-sustaining treatment.

Our analyses disclosed a number of factors associated with time from withdrawing life-sustaining treatment to death. The time was shorter for older patients, male sex, and patients with high CRP on admittance and cerebral hemorrhage. Older patients are frailer, and multiple organ failure probably occurs sooner. High CRP on admittance may signify an infection or other underlying disease, making the patient more prone to multiorgan failure and early death. Cerebral hemorrhage was also associated with shorter time to death after withdrawing life-sustaining treatment than ischemic stroke. A possible explanation is that hemorrhagic stroke tends to be more severe than ischemic stroke. However, after adjusting for stroke severity assessed by the NIHSS score, hemorrhagic stroke was still associated with shorter time to death than ischemic stroke.

In-hospital mortality was low for both patients with ischemic and hemorrhagic stroke compared with other studies. ${ }^{1,6}$ This indicates that the decision to withdraw lifesustaining treatment occurred in patients with severe stroke and very poor prognosis, and it is unlikely that neurologists in our department were overly pessimistic in prognosticating outcome. $^{2}$

Our study has some limitations. The most important limitation is the retrospective identification of time for withdrawing 
life-sustaining treatment. However, a prospective study on this topic is in our opinion unethical, because it is possible that an ongoing, prospective study might influence on the decision of withdrawing life-sustaining treatment or not. Data on CT or MRI immediately prior to this decision were not available for analyses.

\section{Conclusion}

In conclusion, 1 week after withdrawing life-sustaining treatment, a quarter of the patients with severe stroke remained alive in our department. Short time to death was associated with high age, male sex, hemorrhagic stroke, and high CRP on admission.

\section{Disclosure}

The authors have no conflicts of interest.

\section{References}

1. Tveiten A, Ljøstad U, Mygland A, Thomassen L, Pripp AH, Naess H. Intracerebral hemorrhage in southern Norway - a hospital-based incidence study. Eur Neurol. 2012;67(4):240-245.
2. Becker KJ, Baxter AB, Cohen WA, et al. Withdrawal of support in intracerebral hemorrhage may lead to self-fulfilling prophecies. Neurology. 2001;56(6):766-772.

3. Yoneda Y, Uehara T, Yamasaki H, Kita Y, Tabuchi M, Mori E. Hospital-based study of the care and cost of acute ischemic stroke in Japan. Stroke. 2003;34(3):718-724.

4. Creutzfeldt CJ, Holloway RG. Treatment decisions after severe stroke: uncertainty and biases. Stroke. 2012;43(12):3405-3408.

5. Wong KS. Risk factors for early death in acute ischemic stroke and intracerebral hemorrhage: a prospective hospital-based study in Asia. Asian Acute Stroke Advisory Panel. Stroke. 1999;30(11): 2326-2330.

6. Heuschmann PU, Kolominsky-Rabas PL, Misselwitz B, et al; German Stroke Registers Study Group. Predictors of in-hospital mortality and attributable risks of death after ischemic stroke: the German Stroke Registers Study Group. Arch Intern Med. 2004;164(16):1761-1768.

7. Appelros P, Jonsson F, Åsberg S, et al; Riks-Stroke Collaboration. Trends in stroke treatment and outcome between 1995 and 2010: observations from riks-stroke, the Swedish Stroke Register. Cerebrovasc Dis. 2014;37(1):22-29.

8. Organisation WH. Cerebrovascular Disorders. Geneva: World Health Organisation; 1978.

9. Wood GG, Martin E. Withholding and withdrawing life-sustaining therapy in a Canadian intensive care unit. Can J Anaesth. 1995;42(3): 186-191.
Vascular Health and Risk Management

\section{Publish your work in this journal}

Vascular Health and Risk Management is an international, peerreviewed journal of therapeutics and risk management, focusing on concise rapid reporting of clinical studies on the processes involved in the maintenance of vascular health; the monitoring, prevention and treatment of vascular disease and its sequelae; and the involvement of

\section{Dovepress}

metabolic disorders, particularly diabetes. This journal is indexed on PubMed Central and MedLine. The manuscript management system is completely online and includes a very quick and fair peer-review system, which is all easy to use. Visit http://www.dovepress.com/ testimonials.php to read real quotes from published authors. 